\title{
Review of Performance Pay at County Level Public Hospitals
}

\author{
Shuwei Gu ${ }^{1}$, Qiuying Yue ${ }^{1}$, Xiaowen Wan ${ }^{1, a}$ \\ ${ }^{1}$ School of Economics and Management, Jiangxi University of Traditional Chinese Medicine, China
}

\begin{abstract}
Performance pay in county-level public hospitals is an important part of the reform of public hospitals, which largely determines the success or failure of the reform of public hospitals. This paper reviews the concept of performance pay, the recognition of hospitals and staff, the main performance pay model, current situation, design principles and ideas in County-level Public hospitals, and provides some reference for future research and reform direction of performance pay in County-level Public hospitals.
\end{abstract}

In 2015, the General Office of the State Council "opinions on the implementation of comprehensive reform of county-level public hospitals" put forward that "the salary level of medical staff should be reasonably determined... Perfecting the performance pay system, hospitals distribute their income independently through scientific performance appraisal, so as to achieve more work, more merit and better remuneration ${ }^{[1]}$. The promulgation of relevant policies and regulations provides a good external environment for the reform of personnel compensation system in County-level Public hospitals. Based on a systematic review of the status quo of performance pay in county-level public hospitals in various regions, this paper analyzed and summarized the problems existing in the pay system, and put forward an optimization plan for the reform of performance pay system in County-level Public hospitals.

\section{Data and methods}

In this paper, CNKI and Wanfang database are used as data sources to search for "county public hospitals" by advanced retrieval method, which contains different keywords, including "performance", "salary", "income", "treatment", "wage", "personnel distribution system", "satisfaction" and "incentive". Theme is used to accurately search for "county public hospitals". Search time is up to 2018.3.15. After reading the literature topics and abstracts, 68 valid literatures were found.

\section{Research status of performance pay in county public hospitals}

\subsection{Definition of concept}

Performance pay originated from Taylor's differential piecework system, which is a kind of salary system that links the employee's salary with the assessment results. Domestic scholars have also made some explorations. Yuan Zheng[2] believes that performance pay is composed of basic and rewarding performance pay, the former is determined by Social Security Departments and the Ministry of Finance according to objective factors such as local economic development level and post responsibilities, while the latter is linked to individual workload and contribution. Hou Tiantian[3] pointed out that performance pay mainly reflects the actual performance and contribution of employees, and hospitals can distribute independently under the guidance of national policies. Huang Jinzao[4] believes that performance pay is a reward based on the overall results of employee performance appraisal. Generally speaking, it is essentially a salary system based on the results of performance appraisal of employees.

\subsection{Hospital and staff awareness}

Performance pay can play an incentive role for medical staff and improve work efficiency, but to a large extent, it depends on the correct understanding of medical staff. Li Xiaorui[5] surveyed 52 county-level public hospitals in a province and found that 34 hospitals had not yet carried out performance appraisal, accounting for $40 \%$ of the total number of county-level public hospitals in the province. From the hospitals that have implemented the performance pay scheme, staff in different positions have different perceptions of performance pay. Yu Xiaobo[6] found that, in the investigation of county-level public hospitals in Zibo, the recognition of performance pay by clinical medical staff was the highest and that of administrative departments was the lowest. The intensity of clinical front-line work is strong, so there will be some emphasis on performance pay plan. Performance pay is higher than that of other departments, and recognition is correspondingly increased.

a Corresponding author:645479278@qq.com 


\subsection{Main mode}

\subsubsection{Equalitarianism model}

The equalitarianism mode refers to the distribution way directly according to the number of departments. The income gap under this mode can be neglected, similar to the "big pot rice" in the planned economy period, which is common in County-level Public hospitals, such as individual departments of Zhangqiu Traditional Chinese Medicine Hospital [7]. The egalitarianism mode has hurt the enthusiasm of medical staff, and is not conducive to the long-term development of hospitals in the end[8].

\subsubsection{Balance of payments model}

Balance of revenue and expenditure model is commonly used in modern hospitals, such as the total performance accounting of Yudu People's Hospital[9]. Performance pay is based on Department income and expenditure reduction, and then a certain amount of total performance pay is extracted according to the proportion, and then it is redistributed by the department interior[10]. This model links medical staff with hospital interests and helps hospitals save expenses[8-9], but it may also lead medical staff to pursue economic interests one-sidedly while ignoring basic ethics, increase income by prescribing more prescriptions and excessive medical treatment or reduce necessary consumables to reduce expenditure, which is contrary to public welfare characteristics[11-13].

\subsubsection{Workload performance model}

Workload performance model refers to a model based on setting different business scoring criteria according to risk difficulty, working time, labor intensity and other factors of different departments, and making more accurate evaluation of workload of medical staff in different departments by using unified scoring coefficient[9]. It is common in the stage of hospital business development, such as the internal personal performance pay accounting in the Department of Yudu County People's Hospital and the performance allocation scheme implemented by a county-level public hospital in Shanghai in 2015. This model effectively solves the problem of one-sided pursuit of economic indicators and low work enthusiasm, which is conducive to the fairness within the hospital.

\subsubsection{Comprehensive performance mode}

The comprehensive performance model is mainly a distribution model based on comprehensive consideration of workload, professional technology, post title and other factors. It can integrate all kinds of comprehensive factors into the distribution plan of performance pay, which is more systematic and comprehensive. But there are also some drawbacks, such as the difficulty in quantifying some factors, leading to standard deviation and unfair. Hospital should consider carefully when formulating allocation criteria and consult with departments to avoid negative idle work caused by mistaken decision-making.

\subsection{Satisfaction study}

According to the survey on the satisfaction degree of performance pay in county public hospitals, the satisfaction degree of most medical staff to performance pay distribution is very low. Pan Min[17] sampled the county-level public hospitals in Chengdu and Panzhihua, and found that the average of salary and performance dimensions of different departments were less than 3 points (total 5 points). Mo Xinxinxin[18] surveyed 3 sample counties in Guangxi and found that the comparison of remuneration and workload was one of the most unsatisfactory items. Feng Xiqiu[19] found that one of the main reasons is unfair, and the performance pay of departments with "good efficiency" will be higher, and the gap between different hospitals is large.

\subsection{Operational performance study}

At present, the medical staff in county public hospitals are not satisfied with the performance pay distribution scheme, which is also due to the existing problems in the distribution mode to varying degrees. There are three main problems:

Firstly, Performance pay assessment is too concerned about economic indicators[20]. Fully embodies the public welfare is the characteristics of public hospitals, public hospitals at the county level are no exception. However, many public hospitals pay too much attention to economic benefits and neglect social responsibility for their development. Li Xiaorui found that in some hospitals which have carried out performance appraisal, the performance pay of some hospitals still stays on the bill of lading, which seriously deviates from the concept of "patient-centered" service and increases the burden of patients' medical expenses[5].

Secondly, the gap between departments is too small. Only when we have a certain gap can we really play an incentive role. However, the phenomenon of egalitarianism and "big pot" in county public hospitals is common. Pang Jialing found that the performance pay gap is too small, and there is no difference between doing more and doing less, which is one of the reasons for dissatisfaction [21].

Thirdly, Medical staff's pay is out of proportion to their income. Payment in proportion to income can reflect fairness, but the performance distribution of county-level public hospitals can not reflect it well. The different volume of business and workload caused by some objective factors among hospitals will also lead to external unfairness[4]. In hospitals, unreasonable design of performance pay system, inadequate distribution according to work and inadequate consideration of actual working ability are also major reasons[22].

\subsection{Design principles and ideas}

"More work, more pay, better pay" provides the general principle for the performance remuneration system of county-level public hospitals. Based on this principle, Shishi City allocates performance according to 
comprehensive indicators and tilts to the clinical front line[23]; Danyang People's Hospital determines remuneration[24] according to the principles of clinical $>$ Medical Technology $>$ Assistant and functional departments, and according to factors such as quality and quantity of work; Liuyang People's Hospital of Hunan Province determines to the clinical front line, medical quality and key posts. Business backbone and management and efficiency tilt principle[25]. In the long run, "More work, more pay, better pay" will become the general principle of performance pay reform in the future, and realize the transformation from policy to reality.

\section{Evaluation and prospect}

As an important part of the reform of public hospitals, the performance pay of county-level public hospitals is of great importance. However, at present, there are few special studies on performance pay in county public hospitals, most of which are mentioned incidentally in the study of compiling or the whole salary system, or about the overall study of public hospitals, but not to county public hospitals. As a part of public hospitals, county-level public hospitals also have some personality factors in their performance pay. Therefore, it is necessary to study the performance pay of county-level public hospitals rather than incorporate it into the study of public hospitals. In addition, the existing studies are mostly focused on case studies or general issues, but lack of systematic review. The research on systematic review needs to be enriched and excavated.

\section{References}

1. Website of Department of System Reform. Opinions on Implementing Comprehensive Reform of county public hospitals [EB/OL]. (2014-03-26) [2014-04-04]. http://www.nhfpc.gov.cn/tigs/s3581/201404/9204caf 25546 eb875274ba77499e04.shtml.

2. Yuan Zheng. Research on the reform of personnel system and distribution system in public hospitals [J]. Cooperative economy and science and technology, 16(2013).

3. Hou Tiantian. Study on Influencing Factors of doctors'salary in county public hospitals based on job value [D]. Qingdao University, (2015).

4. golden kitchen. Discussion on salary reform of county public hospitals under the new health care reform [J]. contemporary accounting, 04(2016).

5. Li Xiaorui, Yu Runji. Investigation and analysis of the implementation of performance appraisal system in county-level public hospitals [J]. Health Economic Research, 06(2016).

6. Investigation and analysis of performance pay of employees in county-level public hospitals of $\mathrm{Yu}$ Xiaobo, Tian Liqi, Wang Wei. Zibo [J]. Qilu Medical Journal, 30(2015).

7. Zhugeli. Study on the process and effect evaluation of comprehensive reform of county-level public hospitals in Shandong Province [D]. Shandong University, (2017).
8. Zhang Deyu. Research on Performance Wage Distribution Method of County Public Hospital Based on Budget Proportion Method [D]. Nanhua University, (2016).

9. Deng Dasong, Liu Zhenyu. Probe into the Performance Pay Management System of County Public Hospital Based on RBRVS --- Take Yudu County People's Hospital of Jiangxi Province as an example [J]. Journal of Jiangxi University of Finance and Economics, 06(2017).

10. Yin Yanling, Xu Bing and Yang Yan. Discussing the Hospital Bonus Allocation System Based on Workload [J]. Hospital Management Forum, 30(2013)

11. Zhong Wei. Discussion on the transformation of performance reform system in county public hospitals, [J]. enterprise research, 10(2014).

12. Lu Xuemei. A Brief Talk on the Design of Performance Wage Reform in county public hospitals [J]. Finance and Economics (Academic Edition), 23(2016).

13. Jiang Zhengqiang. Research on performance management of Taizhou $\mathrm{H}$ county hospital [D]. Jiangxi University of Finance and Economics, (2016).

14. $\mathrm{Hu}$ Qiongwei. Research on the positive incentive mechanism of a public hospital in Shanghai [D]. Shandong University, (2015).

15. Jiang Zongshun. Reflections on the guiding system of performance pay distribution in hospitals [J]. Hospital Management of China, 33(2013).

16. Zhao Shasha. Discussion on Performance Wage Distribution in Public Medical Institutions [J]. Primary Health Care in China, 27(2013).

17. Pan Min, Huang Maojuan, Zhang Mei. Survey and Suggestions on Occupational Evaluation of Medical Staff in County-level Public Hospitals from the Perspective of Pay Equity [J]. Jiangsu Health Career Management, 27(2016).

18. Moxinxinxin, Feng Qiming, Luo Hongye, Luoping, Chen He, Gao Hongda, Li Yangfeng, Zhangjuan. Analysis of job satisfaction and its influencing factors of medical staff in county-level public hospitals of Guangxi [J]. Modern Chinese Doctors, 53(2015).

19. Feng Xiqiu. Survey and analysis of staff satisfaction with county-level public hospital reform in Anhui Province [D]. Anhui Medical University, (2014).

20. Yang Qunhui. Research on Performance Management of Hospital Organization Based on PDCA Closed Loop [J]. Modern Hospital, 17(2017).

21. Pang Jialing, Wu Weimin, Yang Xiuling, Jiang Shuai and Fan Li. Investigation and analysis of satisfaction of medical staff after the reform of County-level public hospitals [J]. Health Economic Research, 08(2014).

22. Qin Yongfang. New model of personnel compensation system and performance management in county-level public hospitals [J]. China Hospital, 19(2015). 
23. Sui Ping. Research on the establishment and management of county public hospitals [D]. Shandong University, 2017.

24. Zhou Lijian, Lin Yijin. Actively promote the comprehensive reform of county-level public hospitals by taking the opportunity of grade hospital evaluation [J]. Modern Hospital, 16(2016).

25. Deng Zongwei. Practice of economic management and performance distribution in county-level public hospitals under medical reform $[\mathrm{J}]$. Journal of Chinese Medicine Management, 21(2013). 